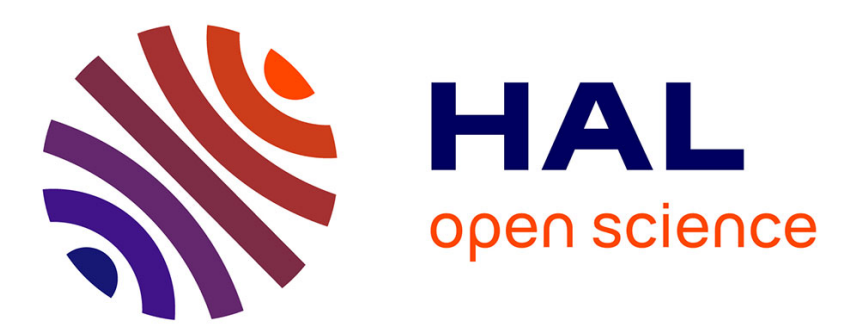

\title{
Etude expérimentale de la libération des conidies d'Erysiphe graminis DC. f. sp. hordei sous l'effet du vent \\ Pierre Pauvert
}

\section{To cite this version:}

Pierre Pauvert. Etude expérimentale de la libération des conidies d'Erysiphe graminis DC. f. sp. hordei sous l'effet du vent. Agronomie, 1984, 4 (2), pp.195-198. hal-00884627

\section{HAL Id: hal-00884627 \\ https://hal.science/hal-00884627}

Submitted on 1 Jan 1984

HAL is a multi-disciplinary open access archive for the deposit and dissemination of scientific research documents, whether they are published or not. The documents may come from teaching and research institutions in France or abroad, or from public or private research centers.
L'archive ouverte pluridisciplinaire HAL, est destinée au dépôt et à la diffusion de documents scientifiques de niveau recherche, publiés ou non, émanant des établissements d'enseignement et de recherche français ou étrangers, des laboratoires publics ou privés. 


\section{Etude expérimentale de la libération des conidies d'Ery- siphe graminis DC. f. sp. hordei sous l'effet du vent}

Pierre PAUVERT

I.N.R.A., Station de Pathologie végétale, Route de Saint-Cyr, F 78000 Versailles Des expériences effectuées sur des pustules isolées d'Erysiphe graminis $\mathrm{f}$. sp. hordei, placées dans une petite
soufflerie ont montré que la libération des spores est passive. Toute présence d'eau bloque la libération. Le
seuil minimal de vent dépend de la longueur des chaînes de conidies de la pustule et donc du nombre de spores
présentes; aussi des périodes suffisamment longues sans vent où la sporulation est continue amènent un
abaissement de ce seuil.
La courbe reliant le nombre de spores libérées à la vitesse du vent a une forme en $S$ plus ou moins redressé
suivant la densité initiale de spores. Les spores arrachées à de fortes vitesses du vent sont immatures et
germent mal. L'humidité relative de l'air environnant la pustule n'exerce aucune action sur la libération. La
corrélation négative existant au champ entre le nombre de spores piégées et l'humidité relative du poste
météorologique s'explique par le mouillage (pluie ou rosée) d'une partie des pustules du couvert végétal.

Mots clés additionnels : Ö̈dium, orge, épidémiologie, spores.

Additional key words : Powdery mildew, barley, epidemiology, spores.

\section{INTRODUCTION}

La « libération » est le phénomène qui permet aux spores de franchir la «couche limite» enveloppant les zones sporifères. Selon la terminologie de HIRST \& SCHEIN, (1965), elle est la première phase de la dissémination, précédant la dispersion et la pollution.

Il est bien établi que la libération des conidies d'Erysiphe graminis DC. n'est possible que lorsque les pustules sont sèches. Le mécanisme est passif et de nature anémophile. D'après HAMMET \& MANNERS (1974), une vitesse du vent de $1 \mathrm{~m} . \mathrm{s}^{-1}$ au niveau des pustules suffirait à en déloger des spores. BAINBRIDGE \& LEGG (1976) soulignent l'effet indirect que peut avoir le vent en agitant les feuilles du couvert végétal.
En fait, il est facile d'observer que, sur une plante infectée par l'oïdium et laissée pendant un temps suffisant en air immobile, le moindre courant d'air ou simplement la gravité suffit à libérer des spores. La détermination de la vitesse minimale du vent nécessaire à la libération et, en général, l'étude de ce phénomène ne peuvent se faire qu'en précisant l'état initial de la pustule. En d'autres termes, la libération ne peut être dissociée de la sporulation ; l'allongement des chaînes de conidies qui en résulte rend les conidies en position terminale de plus en plus facilement libérables.

Diverses études faites sur la sporulation d'E. graminis montrent que la production de conidies est continue, optimale vers $20^{\circ} \mathrm{C}$ (PADY et al., 1969; HAMMET \& MANNERS, 1973 ; PAUVERT, 1976), maximale à 100 p. 100 
d'humidité relative, et que l'intensité lumineuse et la photopériode jouent un rôle peu important (WARD \& MANNERS, 1974).

Le but de la présente étude est de quantifier la libération en fonction de la vitesse du vent et du niveau de sporulation atteint par la pustule.

\section{MATÉRIEL ET MÉTHODES}

\section{A. Matériel végétal}

Les expériences sont faites sur des plantules d'orge de la variété «Hâtif de Grignon», particulièrement sensible à l'oïdium. Les plantules qui ont grandi dans une serre à environ $15^{\circ} \mathrm{C}$, sont inoculées au stade 2 feuilles en secouant modérément au-dessus d'elles des feuilles infectées. La composition raciale de l'inoculum n'est pas déterminéc. L'étude est faite sur de jeunes pustules isolées, mesurant environ $2 \mathrm{~mm}^{2}$, portées par la $2^{\mathrm{c}}$ feuille. Une estimation plus précise de la surface de la pustule est faite en considérant que sa forme est elliptique et en mesurant chaque jour le grand axe et le petit axe à l'aide d'une loupe munie d'un réticule oculaire. Pendant toute la durée de l'expérience, les plantes maintenues en pot sont arrosécs quotidiennement et soumises à un éclairage artificiel d'environ 10000 lux au niveau des feuilles (tubes fluorescents «blanc industrie »), la photophase est de $12 \mathrm{~h}$ sur 24 .

\section{B. Dispositif d'étude de la libération}

La feuille portant la pustule est inséréc transversalement dans une petite soufflerie. Celle-ci (fig. 1) est constituée d'un tube de verre de $1,1 \mathrm{~cm}$ de rayon interne, prolongé par une partie conique. Une ouverture à la partic supéricure de la section cylindrique permet le passage de la feuille qui est maintenue par un petit couvercle assurant l'étanchéité. On utilise de l'air comprimé dont le débit est contrôlé par une vanne et un débitmètre. L'humidité relative est régléc par barbottage de l'air dans un mélange en proportions convenables d'eau et de glycérol. Un contrôle précis de l'humidité relative obtenue ainsi que de la température de l'air est fait en remplaçant la soufflerie par un psychromètre électrique constitué par 2 thermocouples: l'un sec, l'autre humide. Les tensions électriques obtenues sont mesurées à l'aide d'un potentiomètre manuel. Des tables fournissent les valeurs de l'humidité relative en fonction de la température du thermocouple sec et de la température du thermocouple humide.

La vitesse du vent au voisinage de la pustule est estimée en divisant le débit par la section du tube ; cette approximation conduit à une valeur proche de la réalité car les grandes dimensions du tube et les valeurs encore faibles du débit rendent les phénomènes de friction au niveau des parois négligeables. Les spores libérées et emportées par le flux d'air sont capturées à la sortie de la partie conique du tube grâce à une lame porte-objet enduite de lanoline ou sur une plaque de gélose à $2 \mathrm{p}$. 100. Le comptage total des spores recueillies est fait au microscope. Les spores placées sur gélose sont mises à germer dans des boîtes de Petri dont le fond contient un mélange eau-glycérol assurant une humidité relative proche de 98 p. 100 .

\section{Conduite des expériences}

Avant chaque expérience, la pustule mise en place est soumise à l'effet du flux d'air maximal délivré par le système $\left(3,5 \mathrm{~m}^{3} \cdot \mathrm{h}^{-1}\right.$, soit environ $2,5 \mathrm{~m} \cdot \mathrm{s}^{-1}$ au niveau de la pustule), entraînant la libération de toutes les spores détachables à cette vitesse, puis laissée au repos sóus un flux d'air de faible débit (environ $0,1 \mathrm{~m}^{3} \cdot \mathrm{h}^{-1}$ ), pendant des temps variables allant de quelques heures à plusieurs jours. A la fin du temps de repos, la pustule est soumise à des flux d'air de débit croissant : 0,5-1-1,5-2-2,5-3-3,5 $\mathrm{m}^{3} \cdot \mathrm{h}^{-1}$ pendant $1 \mathrm{mn}$. Les spores libérées à chaque débit sont dénombrées et cumulées aux spores déjà libérées aux débits inférieurs.

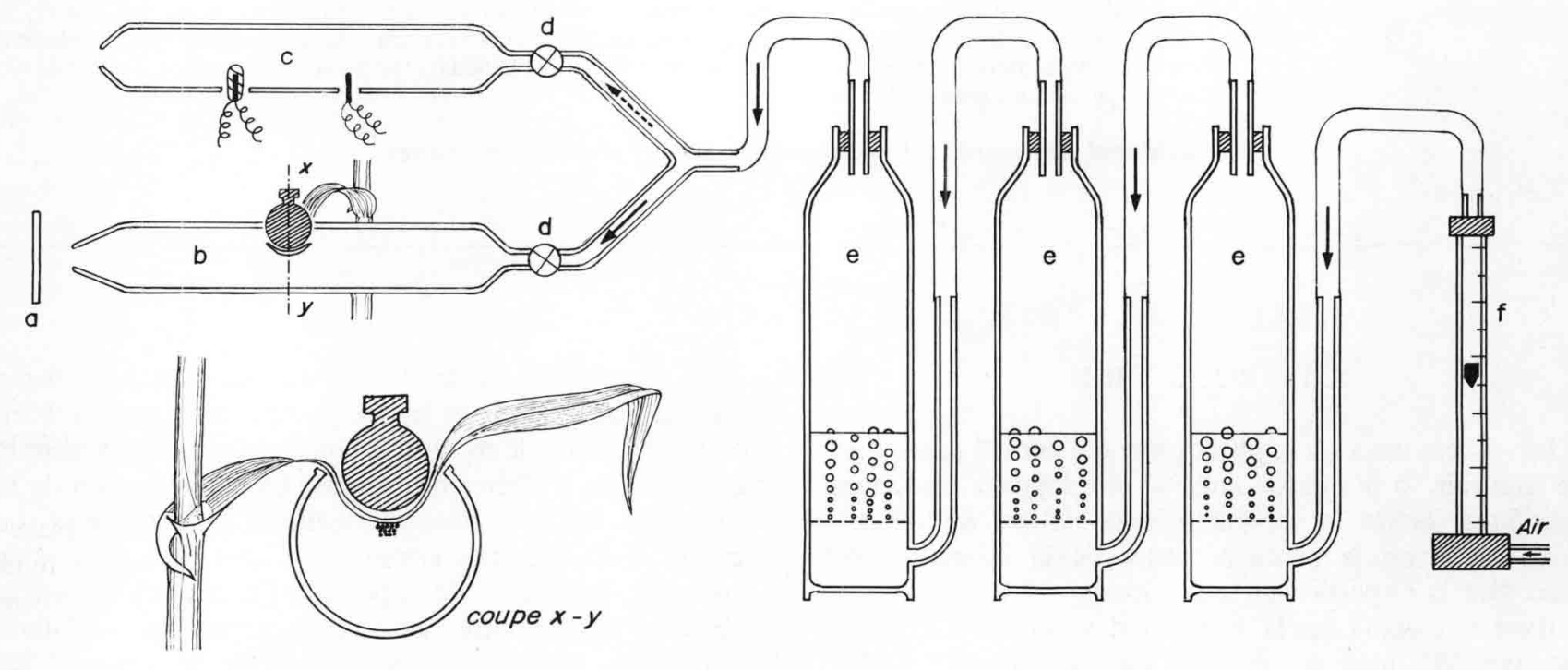

Figure 1

Dispositif d'étude de la libération des conidies par le vent. Wind tunnel apparatus for the study of conidial liberation:

a. Lame de microscope enduite de lanoline; microscope slide coated with lanoline. b. Soufflerie; wind tunnel.

c. Psychromètre électrique; electric psychrometer.

d. Vannes; valves.

e. Flacons laveurs avec mélange eau-glycérine; humidity control.

f. Débitmètre ; flowmeter. 


\section{RÉSULTATS}

\section{A. Influence de la densité initiale de spores dans la pustule (tabl. 1)}

Le seuil de vitesse du vent permettant le début de la libération est d'autant plus bas que la densité de spores dans la pustule est plus élevée (ou en d'autres termes que le temps passé par la pustule en atmosphère calme est plus long). La vitesse minimale permettant une libération nous paraît plus basse que la valeur trouvée par HAMMET \& MANNERS (1974). Comme nous l'avons mentionné plus haut, ce seuil n'a pas grande signification. Il est même en fait nul, puisqu'au bout de $3 \mathrm{j}$ de repos, des spores se libèrent par seule gravité, ce qui semble correspondre approximativement à une densité de 3000 spores $\cdot \mathrm{mm}^{-2}$, si l'on fait l'hypothèse que la sporulation reste inchangéc.

\section{B. Influence de la vitesse du vent}

Les courbes reliant le nombre de spores libérées en fonction de la vitesse du vent (fig. 2) ont une forme en S plus ou moins redressé suivant la densité initiale en spores. Pour une pustule dont le stock de spores est grand, une libération importante se produit pour des vitesses du vent modérées. Au contraire, l'énergie requise pour arracher des spores d'une pustule qui a déjà été soumise à des flux d'air importants ou qui a peu sporulé est plus forte. Ce résultat paraît lié à la disposition en chaînes des spores. La plus grande facilité de libération des conidies en position terminale sur la chaîne peut s'expliquer par la diminution de la solidité du lien qui unit une spore à une autre, mais aussi par le fait que les spores atteignent alors une distance par rapport au plan de la feuille où la vitesse du vent est plus élevée. Le profil de la vitesse du vent en fonction de la distance à la feuille est logarithmique et des chaînes suffisamment longues peuvent même se terminer hors de la couche limite.

\section{Faculté germinative des spores libérées (tabl. 2)}

1) Cas d'une pustule laissée au repos plus de $12 \mathrm{~h}:$ type courbe 1 (fig. 2).

La faculté germinative des spores libérées à une vitesse inférieure à $1 \mathrm{~m} \cdot \mathrm{s}^{-1}$ est bonne, mais chute assez rapidement pour celles qui sont libérées à des vitesses de vent plus importantes.

2) Cas d'une pustule laissée au repos moins de $3 \mathrm{~h}$ : type courbe 4 (fig. 2).

\section{TABLEAU 1}

Influence de la densité initiale de spores de la pustule sur la vitesse minimale de libération.

Relationship between the spore density of the pustule and the minimal liberation speed.

densité de spores : $\left(\right.$ spores $\left.\cdot \mathrm{mm}^{-2}\right)$ spore density

\begin{tabular}{lccccc}
\hline & 110 & 225 & 412 & 660 & 1500 \\
\hline $\begin{array}{l}\text { Vitesse minimale de } \\
\text { libération }\left(\mathrm{m} \cdot \mathrm{s}^{-1}\right)\end{array}$ & 1,1 & 0,7 & 0,45 & 0,2 & $<0,2$ \\
\hline
\end{tabular}

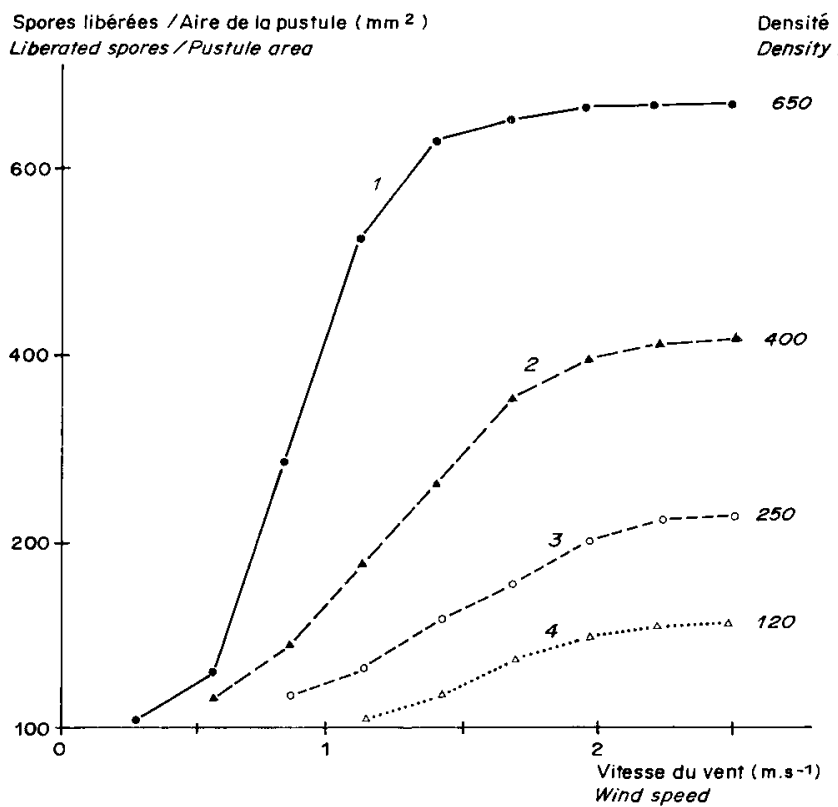

Figure 2

Influence de la densité de spores libérables (spores $\cdot \mathrm{mm}^{-2}$ ) sur la forme des courbes exprimant le nombre de spores libérées par $\mathrm{mm}^{2}$ de pustule en fonction de la vitesse du vent.

Influence of the density of liberable spores in the pustule (spores. $\mathrm{mm}$ ) on the shape of the curve, reflecting the relationships between the number of liberated spores per $\mathrm{mm}^{2}$ and the wind speed.

\section{TABLEAU 2}

Influence du temps de repos de la pustule après épuisement par le vent sur le taux de germination des spores libérées (p. 100).

Relationship between the resting period of the pustule and the germination of the liberated spores.

\begin{tabular}{|c|c|c|c|c|}
\hline \multicolumn{5}{|c|}{ Vitesse du vent : $\mathrm{m} \cdot \mathrm{s}^{-1}$} \\
\hline & $\mathrm{v}<1$ & $1<v<1,7$ & $1,7<v<2,8$ & $v>2,8$ \\
\hline $\begin{array}{l}\text { Pustule au repos } \\
\text { depuis plus de } \\
12 \mathrm{~h}\end{array}$ & 70 & 50 à 65 & 20 à 35 & 5 \\
\hline $\begin{array}{l}\text { Pustule au repos } \\
\text { depuis } 3 \mathrm{~h}\end{array}$ & & 30 & 2 à 8 & 2 \\
\hline
\end{tabular}

La faculté germinative des spores est mauvaise: inférieure à 30 p. 100 pour les spores libérées à des vitesses inférieures à $1,7 \mathrm{~m} \cdot \mathrm{s}^{-1}$ quasi nulle au-dessus.

Ces résultats montrent que les spores aisément détachables par le vent ont une maturité suffisante qui les rend aptes à germer. Ces spores correspondent à la partie terminale de la chaîne.

\section{Influence de l'humidité relative}

Les caractéristiques de la libération des spores ne nous ont pas parc être modifiées par les variations de l'humidité relative. En revanche, la présence d'un film d'eau de condensation gène considérablement la libération. 


\section{CONCLUSION}

Les résultats confirment la passivité du phénomène de libération des spores par le vent. Le fait qu'une augmentation de la vitesse du vent arrache des spores additionnelles indique que dans les conditions du champ, la vitesse instantanée du vent est plus importante que la vitesse moyenne.

Il apparaît contradictoire, à la lumière des résultats obtenus, que l'humidité relative n'exerce aucune influence sur la libération, alors que des piégeages de spores effectués dans des parcelles infectées indiquent que le maximum de spores sont piégées à humidité relative basse. L'explication réside dans le fait que dans toutes les expériences que nous avons faites, bien que l'humidité ait varié, les pustules sont restées sèches. Il n'en est pas de même au champ où les pustules peuvent être mouillées alors que l'humidité relative au niveau du poste météorologique peut être très inférieure à $100 \mathrm{p}$. 100 . Au sein même du couvert végétal, l'humidité relative est plus élevée, en moyenne, au niveau des feuilles inférieures et le séchage des pustules qui s'y trouvent est donc plus long que pour celles des étages supérieurs.

Des vitesses du vent identiques à celles employées dans les expériences précédentes existant dans le microclimat de la pustule correspondraient à des vitesses nettement supérieures au niveau du poste météorologique. En fait, les valeurs ont peu de signification et l'attention doit plutôt être portée sur les «pics» de vent. Une quantification du phénomène de libération dans les conditions naturelles ne peut être faite en ne tenant compte que du vent, puisque l'absence de mouillage des pustules constitue la condition sine qua non à toute libération. Une meilleure connaissance du mouillage du couvert végétal (pluie, rosée...) des céréales et une modélisation du phénomène en fonction des conditions climatiques et culturales s'avère indispensable.

Reçu le 16 mai 1983 Accepté le 22 septembre 1983.

\section{RÉFÉRENCES BIBLIOGRAPHIQUES}

Bainbridge A., Legg B. J., 1976. Release of barley mildew conidia from shaken leaves. Trans. Br. Mycol. Soc., 66 (3), 495-498.

Hammet K. R. W., Manners J. G., 1973. Conidium liberation in Erysiphe graminis. II. Conidial chain and pustule structure. Trans. Br. Mycol. Soc., 61 (1), 121-133.

Hammet K. R. W., Manners J. G., 1974. Conidium liberation in Erysiphe graminis. III. Wind tunnel experiments. Trans. $B r$. Mycol. Soc., 62 (2), 267-282.

Hirst J. M., Schein R. D., 1965. Terminology of infection processes. Phytopathology, 55, 1157.
Pady S. M., Kramer C. L., Clary R., 1969. Sporulation in some species of Erysiphe. Phytopathology, 59, 844-848.

Pauvert P., 1976. Variations quantitatives de la sporulation d'Erysiphe graminis f. sp. hordei. Ann. Phytopathol., 8 (2), 131-140.

Ward S. V., Manners J. G., 1974. Environmental effects on the quantity and viability of conidia produced by Erysiphe graminis. Trans. Br. Mycol. Soc., 62 (1), 119-128. 